\title{
Boron-promoted Deprotonative Conjugate Addition: Development of Geminal diborons as Versatile Soft Pronucleophiles and Acyl An- ion Equivalents
}

\author{
Lucia Wang, Shengjia Lin, Emmanuel Santos, Jenna Pralat, Kaylyn Spotton and Abhishek Sharma*
}

Department of Chemistry and Chemical Biology, Stevens Institute of Technology, Hoboken, NJ, 07030, USA

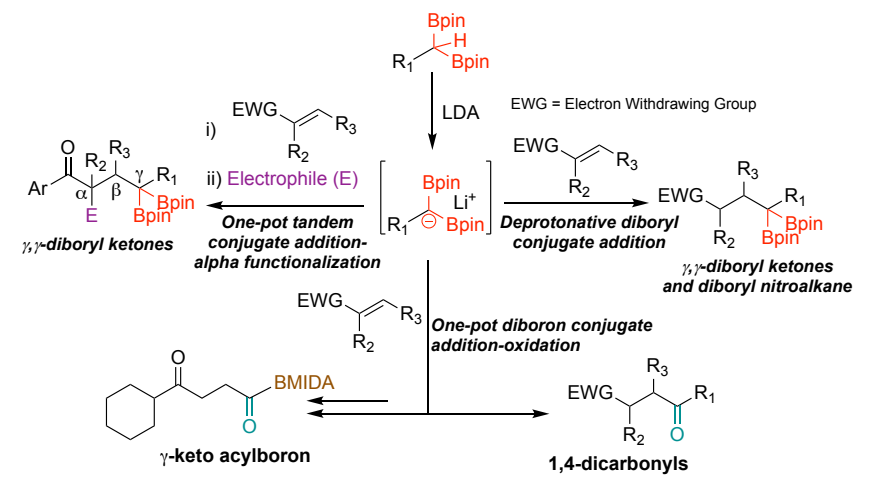

\begin{abstract}
Conjugate addition of $\alpha$-boron stabilized carbanions is an underexplored reaction modality. Existing methods require deborylation of geminal di/triboryl alkanes and/or the presence of additional activating groups. We report the 1,4addition of $\alpha, \alpha$-diboryl carbanions generated via deprotonation of corresponding geminal diborons. The methodology provided a general route to highly substituted and synthetically useful $\gamma, \gamma$-diboryl ketones. The development of geminal diborons as soft pronucleophiles also enabled their use as acyl anion equivalents via one-pot tandem conjugate addition-oxidation sequence.
\end{abstract}

Geminal diborons have emerged as versatile building blocks for preparation of a variety of structurally complex organic compounds. ${ }^{1}$ In comparison to other 1,1-organodimetallic reagents, the geminal organodiborons possess several advantages such as enhanced stability, ease of handling and easy accessibility from abundantly available substrates. Various creative methods have been reported for synthesis and synthetic applications of these reagents. ${ }^{1-3}$ These organoborons can be activated via two complimentary strategies. Treatment of a geminal diboryl alkane with an alkoxide or hydroxide base provides boron stabilized carbanion via monodeborylation. ${ }^{4-6}$ On the other hand, the use of a hindered base leads to $\alpha, \alpha$-diboryl carbanion via deprotonation. ${ }^{1}$ Matteson and others have shown that the $\alpha$-boryl and $\alpha, \alpha$-diboryl carbanions can be used as hard nucleophiles in C-C bond formation with electrophiles via metal catalyzed cross-coupling, nucleophilic substitution or 1,2-addition pathway (Boron-Wittig reaction).1,2,7,8

In contrast to the above mentioned well-established reactivity of $\alpha$-boryl carbanions, the feasibility of 1,4-addition of these nucleophiles is beginning to emerge only recently. Two pioneering reports ${ }^{9,10}$ demonstrated the deborylative conjugate addition of geminal diboryl/triboryl alkanes (Figure 1, a-b). Thereafter, an elegant application of this concept was reported via the 1,4-addition of $C\left(s p^{3}\right)$-carbanions stabilized by boron and allyl groups (Figure 1c).11 In a related study, the Rh catalyzed conjugate addition of $\alpha$-boryl C $\left(s p^{2}\right)$ nucleophiles and its application for synthesis of 1,4-dicarbonyls was disclosed. ${ }^{12}$ Recently, the scope of deborylative conjugate addition was extended to enones ${ }^{13 a}$ (Figure 1d) and vinyl epoxides. ${ }^{13 b}$

Despite the above advances, the full synthetic potential of $\alpha$-boryl carbanion-based conjugate additions remain untapped. For instance, majority of the currently available methods for 1,4-addition of $\alpha$-boryl carbanions involve deborylation of geminal tribory ${ }^{9}$ and diboryl ${ }^{13}$ alkanes besides requiring activation by neighboring benzylic, ${ }^{9}$ allylic $^{11}$ or $\mathrm{Cu}^{10,13}$ groups (Figure 1a-d). In contrast, general strategies for conjugate addition of $\alpha, \alpha$-diboryl stabilized carbanions produced by deprotonation of geminal diborons lacking any other electron-withdrawing groups remain scarce. In view of the tremendous synthetic utility of 1,4-addition reactions, ${ }^{14}$ the development of a diboron-retentive conjugate addition would provide a valuable tool to the emerging chemistry of geminal diboryl alkanes.

In the course of our ongoing work on new synthetic applications of geminal diborons, ${ }^{15-17}$ we became interested to develop a tandem deprotonation-conjugate addition of readily available geminal diboryl alkanes. This approach would preserve the synthetically useful diboron motif in the product while avoiding the installation $/ \mathrm{removal}^{9}$ of an 
Figure 1. Previous approaches for conjugate addition of geminal di/triborons via deborylation (a-d); New deprotonative diboryl conjugate addition and its synthetic applications (e).

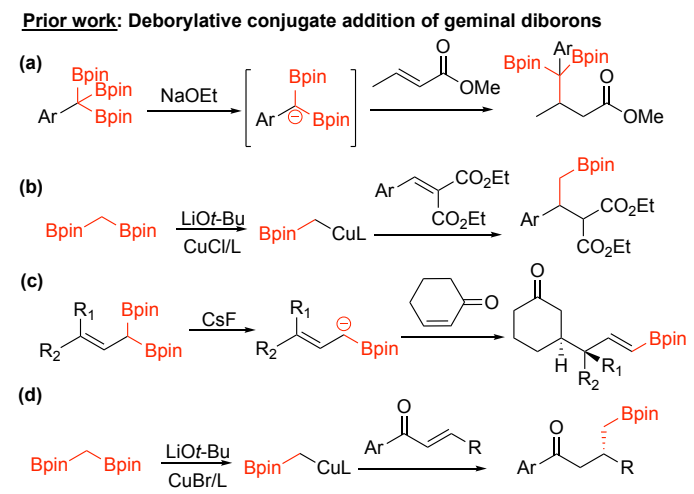

This work: Deprotonative conjugate addition of geminal diborons

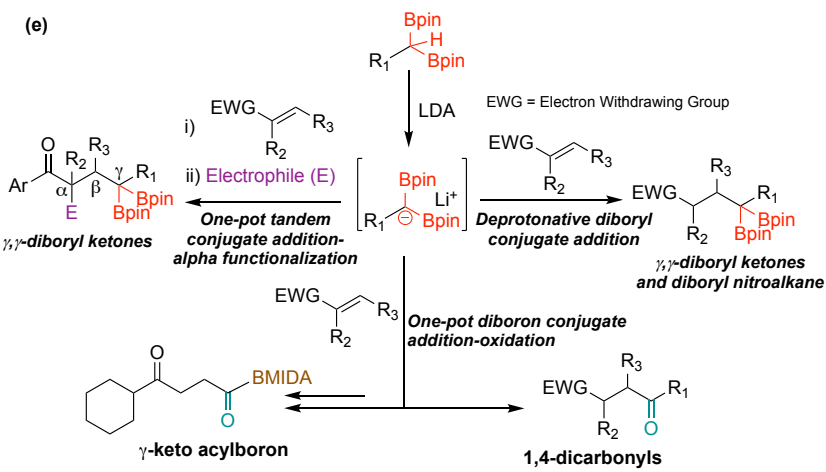

additional boronate group for activating the geminal diboron as soft pronucleophile. Furthermore, the deprotonative 1,4- addition is expected to open-up a general route to synthetically useful and highly functionalized $\gamma, \gamma$-diboryl ketones that would be difficult to prepare using conventional methods. Existing methods ${ }^{18}$ to access these diboryl ketones involve mainly unsubstituted geminal diborons, require expensive catalyst or limit the installation of functional group diversity at the $\alpha$ - and $\beta$ - positions. Herein, we describe a rapid and convenient diboron-retentive conjugate addition reaction of $\alpha, \alpha$-diboryl pronucleophiles and its application to access a wide variety of $\gamma, \gamma$-diboryl ketones (Figure 1e). This strategy also led to the development of geminal diborons as masked acylanion equivalents via onepot conjugate addition-oxidation to provide the valuable 1,4-dicarbonyl frameworks.

We began our studies on the feasibility of deprotonative conjugate addition of diborylalkanes by treating 1a with LDA in THF at $0{ }^{\circ} \mathrm{C}$ followed by the addition of enone $2 \mathbf{a}$ to the same pot. We were pleased to isolate the desired conjugate addition product $\mathbf{3 a}$ in $55 \%$ yield (entry 1, Table 1 ). TLC monitoring of the reaction showed that the enone was consumed within 3-5 min. A screen of the reaction conditions revealed that maintaining a low temperature $\left(0{ }^{\circ} \mathrm{C}\right)$ during the reaction of enone with deprotonated $1 \mathbf{1 a}^{\prime}$ and quenching the reaction mixture after $5 \mathrm{~min}$ improved the yield (entry 2). A further lowering of the reaction temperature to $-20{ }^{\circ} \mathrm{C}$ or increasing the amount of LDA (entries 3-4) diminished the reaction efficiency. Replacing THF with
Table 1. Optimization of 1,4-conjugation addition conditions.

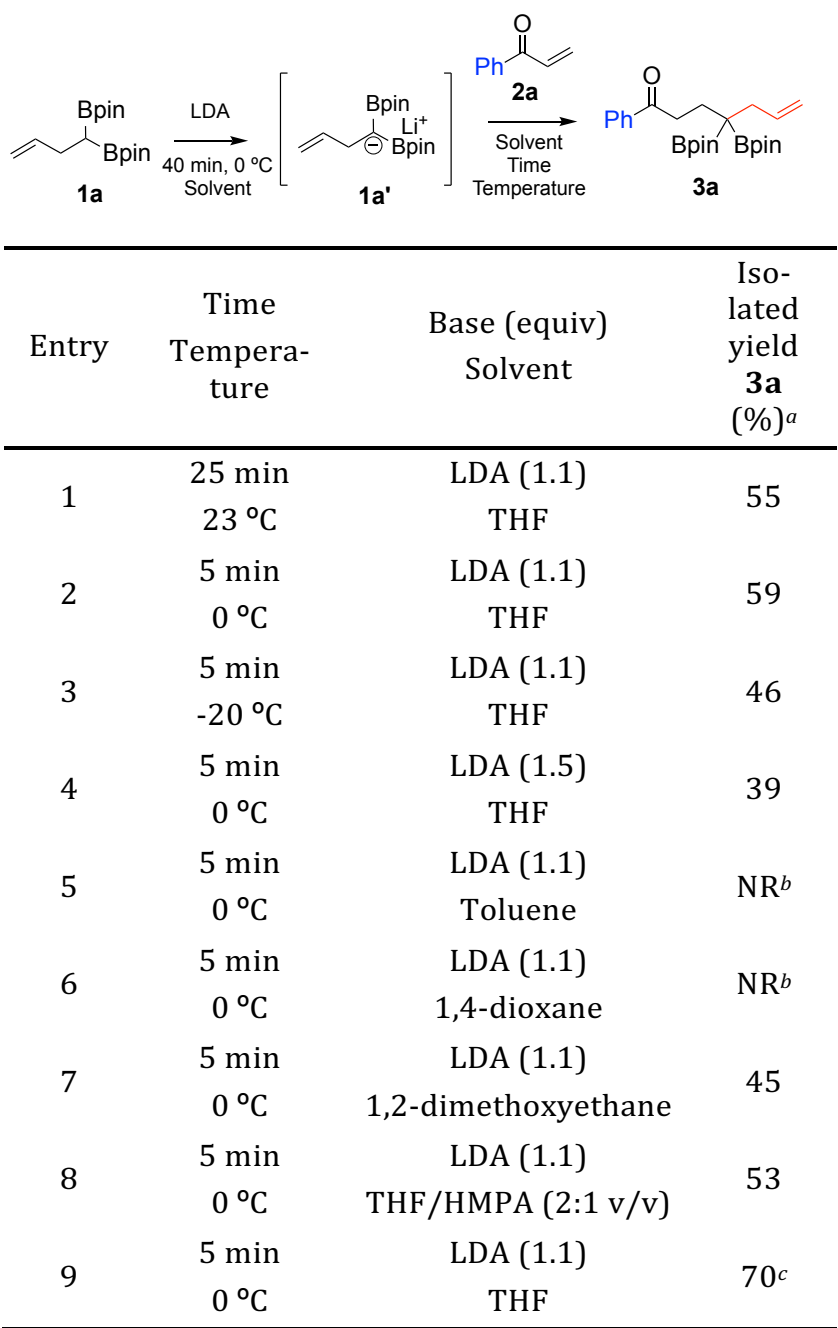

$a$ Isolated yield after aqueous work-up and silica gel column chromatography. ${ }^{b}$ No reaction observed based on TLC analysis. cIsolated yield after quenching with methanol followed by silica gel column chromatography.

other solvents or using HMPA as additive was not beneficial (entries 5-8). The yield of 3a was improved to $70 \%$ by quenching the reaction wth methanol and avoiding aqueous workup (entry 9).

The above optimized conditions were used to investigate the scope of the reaction. Geminal diborons having alkene (Scheme 1, 3a and 3e), alkyl (3d), alkyne (3c), aromatic (3h, $\mathbf{3 k}, \mathbf{3 l}$ ) and heteroaromatic groups (3j) were amenable to the developed protocol. Significantly, the reaction was not limited to geminal diborons as the unsubstituted bis[(pinacolato)boryl] methane also underwent conjugate addition to enone after deprotonation with LDA (3b). To evaluate the feasibility of chemoselective generation and reaction of $\alpha$ boryl stabilized soft nucleophiles from multiboryl alkanes, we prepared the 1,1,2-triboryl alkane (1i) that can undergo LDA-mediated deprotonation at the geminal diboron and/or the $\alpha$-boryl benzylic carbon. Reaction of $\mathbf{1 i}$ with the enone 2a exclusively provided the $\gamma, \gamma, \delta$-triboryl ketone $\mathbf{3 i}$ indicating the preferential deprotonation and 1,4-addition of geminal diboryl anion. The above result and the recently 

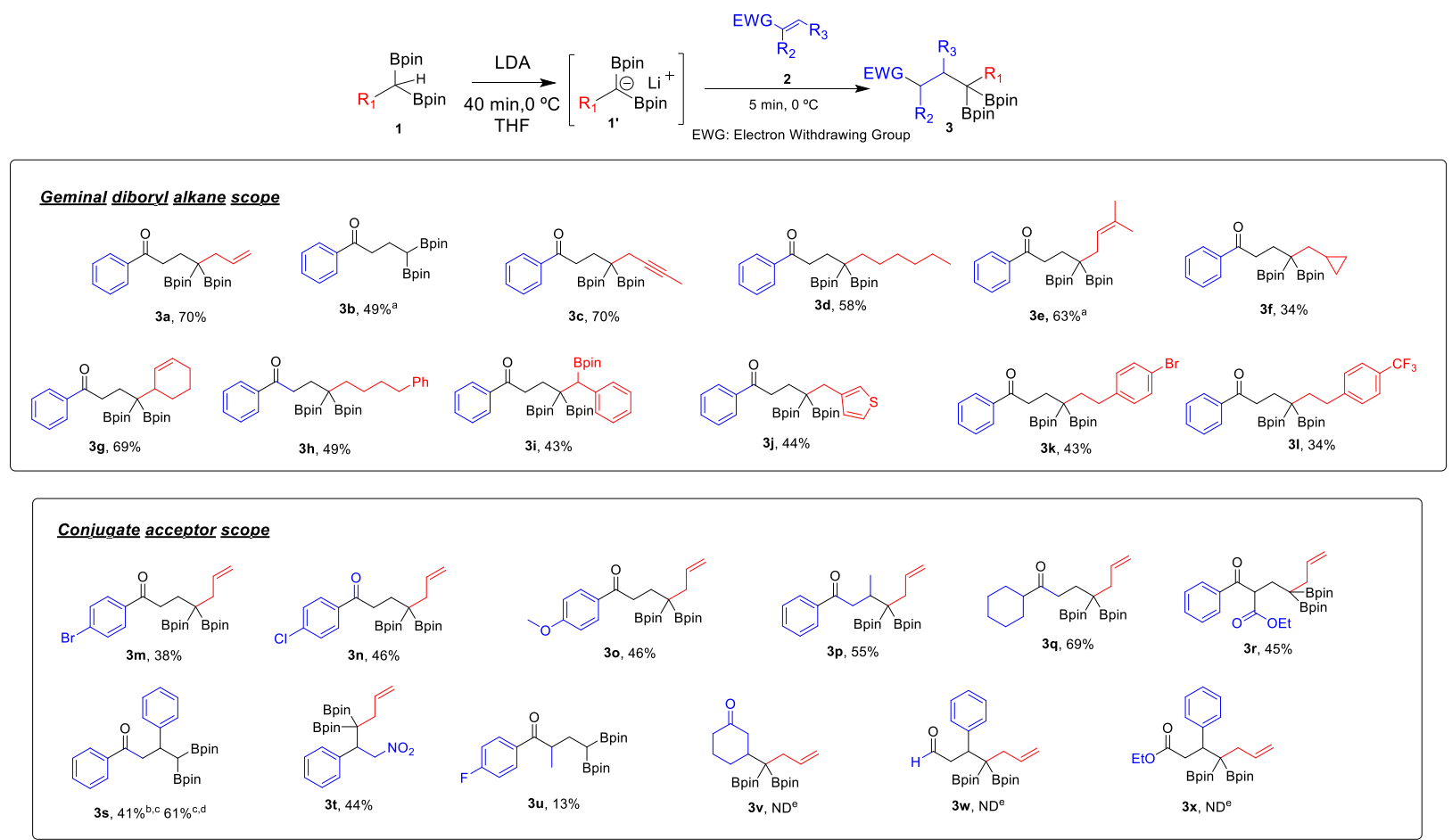

Reaction conditions: 1 (1 equiv.), 2 (1 equiv.), LDA (1.1 equiv.), THF. All reactions were conducted at 0.6 mmol scale unless otherwise specified. ${ }^{a}$ Average yield of three independent experiments. ${ }^{b}$ Isolated yield at $0.6 \mathrm{mmol}$ scale. $c$ The conjugate addition step was conducted at $-30{ }^{\circ} \mathrm{C} .{ }^{d}$ Average yield of two independent experiments at $1.9 \mathrm{mmol}$ scale. $e 1,4$-conjugate addition product was not detected; the 1,2-addition products (via Boron-Wittig reaction) were observed instead

reported deborylative conjugate addition of benzylic triborons ${ }^{9}$ collectively suggest that in comparison to the benzylic group the presence of an additional boryl group provides greater soft nucleophilic character to the $\alpha$-boryl stabilized anion. Multiborylated alkanes such as 1,1,2-tris-borons ${ }^{19}$ have been previously shown to be valuable building blocks for site- selective installation of molecular diversity via sequential transformations of boryl groups. The successful conjugate addition of $\mathbf{3} \mathbf{i}$ allows access to multifunctional tris-borons having ketones as additional synthetic handles.

Next, we evaluated the ability of other conjugate acceptors to participate in deprotonative 1,4-addition. A variety of $\alpha, \beta$-unsaturated ketones bearing aromatic and aliphatic substituents were found to be compatible with the deprotonative conjugate addition of diboryl alkanes. Cyclohexyl enone provided the desired product $\mathbf{3 q}$ in $69 \%$ yield (Scheme 1) while aromatic enones having electron-donating or withdrawing groups were also competent reaction partners (3m-3o). Notably, substituents at the $\alpha$ - or $\beta$ position of enones were tolerated (3p, 3r, 3s, 3u). The reaction was succesfully scaled up to $1.9 \mathrm{mmol}$ and an increase in yield was observed at this larger scale (3s). The developed protocol was also compatible with nitroalkenes as conjugate acceptors (3t). This reaction provided rare $\gamma, \gamma$-diboryl nitroalkanes which can be elaborated into other useful scaffolds. ${ }^{20}$ Cyclic enones, $\alpha, \beta$-unsaturated aldehydes and esters were not compatible substrates as they provided the 1,2-addition product (Boron-Wittig reaction, $\mathbf{3 v}-\mathbf{3} \mathbf{x}$ ) ${ }^{7}$ The above results highlight the complimentary nature of deprotonative conjugative addition (Scheme 1) to the deborylative ${ }^{9,13}$ 1,4-addition of geminal diboron pronucleophiles which was reported to be applicable to mainly enoates $^{6}$ or $\beta$-substituted enones and unsubstituted ${ }^{10}$ geminal diborons (Figure 1a-d).

The developed approach also allowed late-stage installation of additional structural diversity (Scheme 2). For instance, the enolates resulting from the deprotonative

Scheme 2. Scope of tandem conjugate addition followed by enolate trapping.

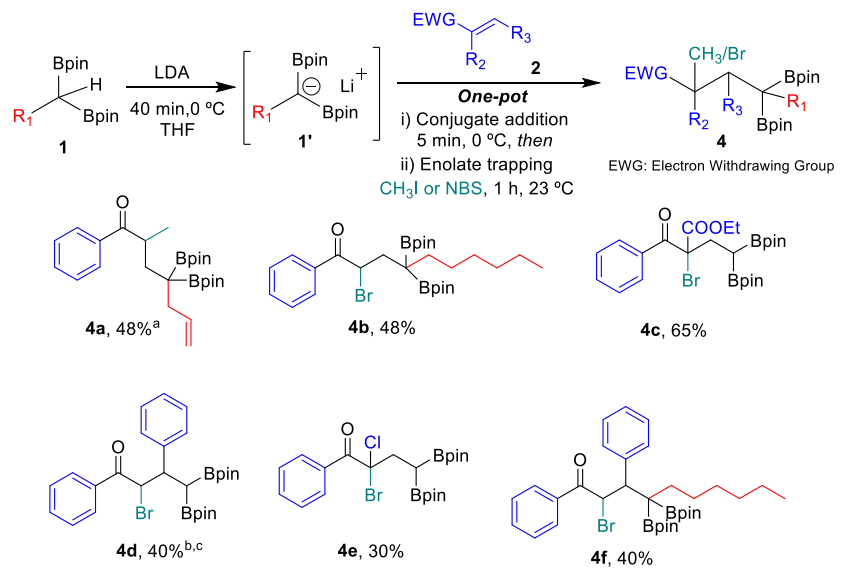

Reaction conditions: 1 ( 1 equiv.), 2 (1 equiv.), LDA (1.1 equiv.), THF; then electrophile (MeI 3 equiv. or NBS 1.1 equiv.) was added and stirred for $1 \mathrm{~h}$ at $23{ }^{\circ} \mathrm{C}$. aUpon conjugate addition completion, HMPA ( $2 \mathrm{~mL}$ ) was added. $b$ Combined yield of two diastereomers. $c$ The conjugate addition reaction was conducted at $-30{ }^{\circ} \mathrm{C}$. 
addition of geminal diborons were successfully quenched with electrophiles in the same pot. This strategy allowed access to highly functionalized $\gamma, \gamma$-diboryl ketones (4a-f) including scaffolds having geminal halo-ester $4 c$, vicinal haloaryl (4d) or geminal dihalo groups (4e) which would be difficult to install using the existing methods. ${ }^{18}$ Interestingly, the tandem conjugate addition and $\alpha$-functionalization of $\beta$ substituted enone using unsubstituted geminal diboron and NBS provided a mixture of two diastereomeric products (4d) while the use of substituted geminal diboryl alkane as Michael donor afforded only one diastereomer (4f).

To further expand the synthetic utility of geminal diboryl stabilized carbanions, we envisioned their use as acyl anion equivalents. There has been considerable interest in development of new types of umpolung reagents due to their utility in accessing carbonyl compounds having 1,4 functional group relationships. ${ }^{21,22}$ Given the facile reactivity of $\mathbf{1}$ as soft nucleophiles (Scheme 1-2) and the mild conditions required for oxidation of boronate group,12, 15 we hypothesized that a one-pot conjugate addition-diboron oxidation sequence could be feasible. The resulting 1,4-dicarbonyl products are privileged intermediates for synthesis of bioactive compounds. ${ }^{23}$ To this end, the deprotonated diboron $\mathbf{1}^{\prime}$ was treated with enone 2 followed by the addition of $\mathrm{NaBO}_{3}$ to the same pot (Scheme 3 ). The above tandem reaction succesfully provided the desired 1,4-diketone $\mathbf{5}$. The protocol was compatible with geminal diboryl alkanes having saturated $(\mathbf{5 d}, \mathbf{5 i}, \mathbf{5 e})$, unsaturated $(\mathbf{5 a}, \mathbf{5 b})$ and heteroaromatic groups (5c).

\section{Scheme 3. Scope of one-pot diboron conjugate addition-} oxidation.

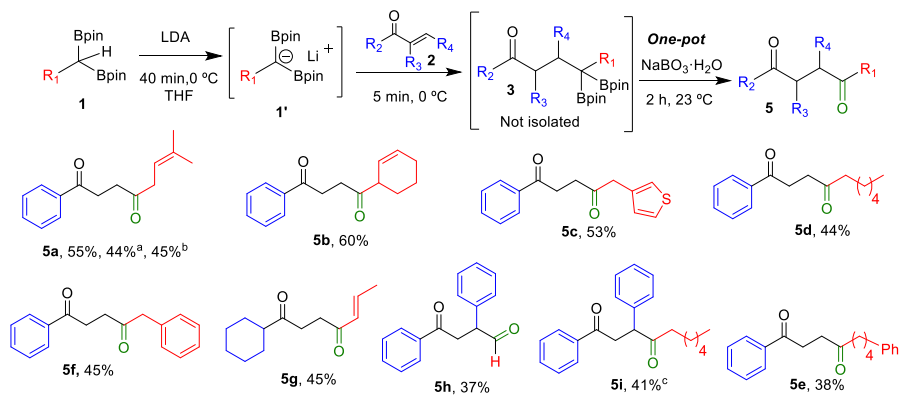

Reaction conditions: 1 ( 1 equiv.), 2 ( 1 equiv.), LDA ( 1,1 equiv.), THF; then $\mathrm{NaBO}_{3} \cdot \mathrm{H}_{2} \mathrm{O}$ (1.3 equiv.), $\mathrm{H}_{2} \mathrm{O}, 2 \mathrm{~h}, 23{ }^{\circ} \mathrm{C}$. All reactions were performed at $0.6 \mathrm{mmol}$ scale unless otherwise specified. ${ }^{a}$ Isolated yield after 2-pot protocol. ${ }^{b}$ Isolated yield after 2.6 mmol scale reaction. ${ }^{c} \mathrm{H}_{2} \mathrm{O}_{2}\left(2 \mathrm{~mL}, 30 \%\right.$ solution in $\left.\mathrm{H}_{2} \mathrm{O}\right)$ and $\mathrm{NaOH}\left(2 \mathrm{~mL}, 2 \mathrm{M}\right.$ solution in $\mathrm{H}_{2} \mathrm{O}$ ) were used instead of $\mathrm{NaBO}_{3} \cdot \mathrm{H}_{2} \mathrm{O}$.

The geminal diboron framework of 1,1,2-trisboryl alkane $\mathbf{1 i}$ also participated in the desired one-pot reaction while its benzylic boronate component underwent protodeborylation under the oxidation conditions (5f). Interestingly, the tandem conjugate addition-diboron oxidation of $\mathbf{1 a}$ and $\mathbf{2 f}$ afforded the conjugated 1,4-diketone via isomerization of terminal olefin (5g). Furthermore, unsubstituted diboron could be used thereby providing $\alpha$-substituted 1,4ketoaldehydes (5h) which represents one of the more challenging24 1,4-dicarbonyls. The benefit of the one-pot conjugate addition-oxidation was evaluated by conducting the corresponding two-pot reaction sequence involving isolation of $\gamma, \gamma$-diboryl ketone (3e) followed by its oxidation.
This two-pot approach provided lower yield (44\%) of the 1,4-diketone (5a, Scheme 3) in comparison to the one-pot protocol (55\%). Finally, the one-pot reaction was successfully scaled up to $2.6 \mathrm{mmol}$ without a significant decrease in reaction yield (5a, 45\%, Scheme 3 ).

To investigate the utility of the diboryl conjugate additionoxidation approach in enabling access to rare 1,4-dicarbonyls, we became interested in developing a synthetic route to $\gamma$-keto acylborons (Scheme 4). The unique chemical reactivity and applications of acylborons in organic synthesis and bioconjugation have inspired several creative synthetic methods. ${ }^{25},{ }^{26}$ While the $\alpha$-keto ${ }^{27}$ and $\beta$-keto acylborons $^{28}$ can be prepared using several approaches, methods to access $\gamma$-keto acylborons are scarce. ${ }^{29}$ Inspired by our recently developed route to MIDA acylborons, ${ }^{15,16}$ the geminal diboron motif in the conjugate addition product $\mathbf{3 y}$ (Scheme 4) was successfully transformed into the unsymmetrical diboron 6. Chemoselective oxidation ${ }^{15}$ of 6 provided the desired $\gamma$-keto MIDA acylboron 7 in 34\% yield. In view of the ready availability of $\alpha$ - and $\beta$-substituted $\gamma, \gamma$-diboryl ketones (Scheme 1-2), the above chemoselective oxidation approach is expected to enable a general route to $\gamma$-keto acylborons.

\section{Scheme 4. Synthesis of $\gamma$-keto acylboron via conjugate addition-oxidation sequence.}

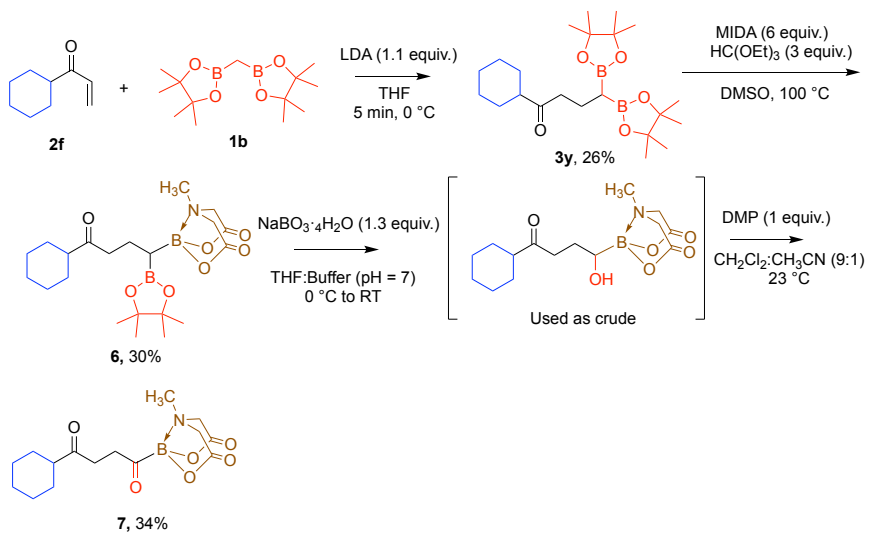

In conclusion, the diboron-retentive conjugate addition of geminal diboryl alkanes with enones and nitroolefin has been developed for the first time. The methodology is applicable on both terminal and internal enones as conjugate acceptors while $\alpha$-substituents on these substrates are also tolerated. A variety of substituted as well as unsubstituted geminal diborons were compatible reaction partners and installation of additional functional group diversity was feasible by trapping the enolate with electrophiles. This reaction afforded a general route to highly substituted and synthetically useful $\gamma, \gamma$-diboryl ketones. Furthermore, geminal diborons were shown to be effective acyl anion equivalents via a one-pot conjugate addition-oxidation sequence. Collectively, these studies provide geminal diboryl alkanes as versatile soft pronucleophiles for rapid $\mathrm{C}-\mathrm{C}$ bond formation with enones and nitroolefins and eliminate the requirement of installing/removing an additional geminal boronate group for activating the pronucleophile towards 1,4addition. 


\section{ASSOCIATED CONTENT}

\section{Supporting Information}

Experimental procedures, characterization data, NMR spectra (PDF).

\section{AUTHOR INFORMATION}

\section{Corresponding Author}

Abhishek Sharma - Department of Chemistry and Chemical Biology, Stevens Institute of Technology, Hoboken, NJ, 07030, USA. E-mail: abhishek.sharma@stevens.edu

\section{Authors}

Lucia Wang - Department of Chemistry and Chemical Biology, Stevens Institute of Technology, Hoboken, NJ, 07030, USA. Shengjia Lin - Department of Chemistry and Chemical Biology, Stevens Institute of Technology, Hoboken, NJ, 07030, USA. Emmanuel Santos - Department of Chemistry and Chemical Biology, Stevens Institute of Technology, Hoboken, NJ, 07030, USA.

Jenna Pralat - Department of Chemistry and Chemical Biology, Stevens Institute of Technology, Hoboken, NJ, 07030, USA.

Kaylyn Spotton - Department of Chemistry and Chemical Biology, Stevens Institute of Technology, Hoboken, NJ, 07030, USA.

\section{Author contributions}

The manuscript was written through contributions of all authors. All authors have given approval to the final version of the manuscript.

\section{Notes}

The authors declare no competing financial interest.

\section{ACKNOWLEDGMENT}

Support for this research from NIH-NIGMS (R35GM143091 and R15GM135891) and Stevens Institute of Technology is gratefully acknowledged. We thank Dr. Athula Attygalle and Ishira Samarasinghe for HRMS analysis

\section{REFERENCES}

(1) (a) Nallagonda, R.; Padala, K.; Masarwa, A. gem-Diborylalkanes: recent advances in their preparation, transformation and application. Org. Biomol. Chem. 2018, 16, 1050-1064. (b) Wu, C.; Wang, J. Geminal bis(boron) compounds: Their preparation and synthetic applications. Tetrahedron Lett. 2018, 59, 2128-2140. (c) Miralles, N.; Maza, R. J.; Fernandez, E. Synthesis and Reactivity of 1,1-Diborylalkanes towards C-C Bond Formation and Related Mechanisms. Adv. Synth. Catal. 2018, 360, 1306-1327. (d) Kim, K. D.; Lee, J. H. Development of Transition-Metal-Free Carbon-Carbon and Carbon-Boron Bond-Forming Reactions by Utilizing 1,1-Bis[(Pinacolato)Boryl]Alkanes. Bull. Korean Chem. Soc. 2018, 39, 5-7. (e) Matteson, D. S.; Moody, R. J. Organometallics, 1982, 1, 20-28.

(2) Babu, K. N.; Massarwe, F.; Reddy, R. R.; Eghbarieh, N.; Jakob, M.; Masarwa, A. Unsymmetrical 1,1-Bisboryl Species: Valuable Building Blocks in Synthesis. Molecules 2020, 25, 959-988.

(3) Lee, J. C. H.; McDonald, R.; Hall, D. G. Enantioselective preparation and chemoselective cross-coupling of 1,1-diboron compounds. Nature Chem. 2011, 3, 894-899.

(4) Hong, K.; Liu, X.; Morken, J. P. Simple Access to Elusive a-Boryl Carbanions and Their Alkylation: An Umpolung Construction for Organic Synthesis. J. Am. Chem. Soc. 2014, 136, 10581-10584.

(5) Potter, B.; Szymaniak, A. A.; Edelstein, E. K.; Morken, J. P. Nonracemic Allylic Boronates through Enantiotopic-Group-Selective
Cross-Coupling of Geminal Bis(boronates) and Vinyl Halides. J. Am. Chem. Soc. 2014, 136, 17918-17921.

(6) Endo, K.; Ohkubo, T.; Hirokami, M.; Shibata, T. Chemoselective and Regiospecific Suzuki Coupling on a Multisubstituted sp3-Carbon in 1,1-Diborylalkanes at Room Temperature. J. Am. Chem. Soc. 2010, 132, 11033-11035.

(7) Cuenca, A. B.; Fernandez, E. Boron-Wittig olefination with gembis(boryl)alkanes. Chem. Soc. Rev. 2021, 50, 72-86.

(8) Coombs, J. R.; Zhang, L.; Morken, J. P. Synthesis of Vinyl Boronates from Aldehydes by a Practical Boron-Wittig Reaction. Org. Lett. 2015, 17, 1708-1711.

(9) Palmer, W. N.; Zarate, C.; Chirik, P. J. Benzyltriboronates: Building Blocks for Diastereoselective Carbon-Carbon Bond Formation. J. Am. Chem. Soc. 2017, 139, 2589-2592.

(10) Jang, W. J.; Yun, Y. Catalytic Asymmetric Conjugate Addition of a Borylalkyl Copper Complex for Chiral Organoboronate Synthesis. Angew. Chem. Int. Ed. 2019, 58, 18131-18135.

(11) Liang, M. Z.; Meek, S. J. Synthesis of Quaternary Carbon Stereogenic Centers by Diastereoselective Conjugate Addition of BoronStabilized Allylic Nucleophiles to Enones. J. Am. Chem. Soc. 2020, 142, 9925-9931.

(12) Liang, M. Z.; Meek, S. J. Catalytic Enantioselective Synthesis of 1,4-Keto-Alkenylboronate Esters and 1,4-Dicarbonyls. Angew. Chem. Int. Ed. 2019, 58, 14234-14239.

(13) (a) Kim, C.; Roh, B.; Lee, H. G. Restoration of catalytic activity by the preservation of ligand structure: $\mathrm{Cu}$-catalysed asymmetric conjugate addition with 1,1-diborylmethane. Chem. Sci. 2021, 12, 3668-3673. (b) Gava, R.; Fernandez, E. Selective C-C Coupling of Vinyl Epoxides with Diborylmethide Lithium Salts. Chem. Eur. J. 2019, 25, 8013-8017.

(14) Zheng, K.; Liu, X.; Feng, X. Recent Advances in Metal-Catalyzed Asymmetric 1,4-Conjugate Addition (ACA) of Nonorganometallic Nucleophiles. Chem. Rev. 2018, 118, 7586-7656.

(15) Lin, S.; Wang, L.; Aminoleslami, N.; Lao, Y.; Yagel, C.; Sharma, A. A modular and concise approach to MIDA acylboronates via chemoselective oxidation of unsymmetrical geminal diborylalkanes: unlocking access to a novel class of acylborons. Chem. Sci. 2019, 10, 4684-4691.

(16) Lin, S.; Wang, L.; Sharma, A. Acrylic boronate: a multifunctional C3 building block for catalytic synthesis of rare organoborons and chemoselective heterobifunctional ligations. Chem. Sci. 2021, 12, 7924-7929.

(17) Wang, L.; Lin, S.; Zhu, Y.; Ferrante, D.; Ishak, T.; Baba, Y.; Sharma, A. a-Hydroxy boron-enabled regioselective access to bifunctional halo-boryl alicyclic ethers and a-halo borons. Chem. Commun. 2021, 57, 4564-4567.

(18) Zhao, B.; Li, Z.; Wu, Y.; Wang, Y.; Qian, J.; Yuan, Y.; Shi, Z. An Olefinic 1,2-Boryl-Migration Enabled by Radical Addition: Construction of gem-Bis(boryl)alkanes. Angew. Chem. Int. Ed. 2019, 58, 9448-9452.

(19) Salvadó, O.; Fernández, E. Tri(boryl)alkanes and Tri(boryl)alkenes: The Versatile Reagents. Molecules 2020, 25, 1758-1775.

(20) Ballini, R.; Palmieri, A. Synthetic Procedures for the Preparation of Nitroalkanes. Adv. Synth. Catal. 2018, 360, 2240-2266.

(21) Denmark, S. E.; Cullen, L. R. Catalytic conjugate addition of acyl anion equivalents promoted by fluorodesilylation. Org. Lett. 2014, $16,70-73$.

(22) Wilde, M. M. D.; Gravel, M. Bis(amino)cyclopropenylidenes as organocatalysts for acyl anion and extended umpolung reactions. Angew. Chem. Int. Ed. 2013, 52, 12651-12654.

(23) Kaldre, D.; Klose, I.; Maulide, N. Stereodivergent synthesis of 1,4-dicarbonyls by traceless charge-accelerated sulfonium rearrangement. Science 2018, 361, 664-667.

(24) García-Santos, W.H.; Mateus-Ruiz, J. B.; Cordero-Vargas, A. Visible-Light Photocatalytic Preparation of 1,4-Ketoaldehydes and 1,4-Diketones from a-Bromoketones and Alkyl Enol Ethers. Org. Lett. 2019, 21, 4092-4096. 
(25) Šterman, A.; Sosič, I.; Gobec, S.; Časar, Z. Recent Advances in the Synthesis of Acylboranes and Their Widening Applicability. ACS Omega 2020, 5, 17868-17875.

(26) Wu, D.; Taguchi, J.; Tanriver, M.; Bode, J. W. Synthesis of Acylboron Compounds. Angew. Chem. Int. Ed. 2020, 59, 16847-16858. (27) He, Z.; Trinchera, P.; Adachi, S.; St Denis, J. D.; Yudin, A. K. Oxidative Geminal Functionalization of Organoboron Compounds. Angew, Chem. Int. Ed. 2012, 51, 11092-11096.

(28) Ivon, Y. M.; Mazurenko, I. V.; Kuchkovska, Y. O.; Voitenko, Z. V.; Grygorenko, 0. O. Formyl MIDA Boronate: C1 Building Block Enables Straightforward Access to a-Functionalized Organoboron Derivatives. Angew. Chem. Int. Ed. 2020, 59, 18016-18022.
(29) Michalland, J.; Zard, S. Z. A Convergent, Stereoselective Route to Trisubstituted Alkenyl Boronates. Org. Lett. 2021, 23, 80188022. 